\title{
Dental Management of Ectodermal Dysplasia: A Report of Two Cases
}

\author{
Rajeev Pandey ${ }^{1}$, Anit Khatri ${ }^{2}$ \\ ${ }^{1}$ Department of Oral and Maxillofacial Surgery, ITS Centre for Dental Studies and Research, Muradnagar, Ghaziabad, Uttar Pradesh, \\ India, ${ }^{2}$ Department of Pediatrics and Preventive Dentistry, Pacific Dental College and Hospital, Udaipur, Rajasthan, India
}

Email for correspondence: rajeevpandeyleo@gmail.com

\begin{abstract}
Ectodermal dysplasia (ED) is a rare genetic disorder recognized by the failure of the development of two or more ectodermal structures mainly skin, hair, nails, exocrine glands, and teeth. The main two types of ED are ahydrotic or hypohidrotic and hidrotic ED. It is characterized by three " $\mathrm{H}$ ": Hypotrichosis, hypohidrosis, and hypodontia. Dental problems include a partial or complete absence of primary and/or permanent dentition. Dental management of affected individuals is a very complex issue and a multidisciplinary approach is recommended. The unesthetic appearance, poor self-image, and school/job-related discrimination have a negative psychological effect on the patient. Therefore, an early treatment of craniofacial features is recommended. The management of the orofacial disfigurement provides the patient confidence and improved psychosocial behavior with positive feedback. Dental management of two hypohidrotic type ED with hypodontia is illustrated in the present paper. Both the patients had no familial history of ED. Considering the age of the patients, oral rehabilitation with complete dentures was done and follow-up plan was explained to the parents. Classification, clinical features, etiology, diagnosis, and treatment modalities are also discussed.
\end{abstract}

Key words: Denture, ectodermal dysplasia, hypodontia, syndrome

\section{INTRODUCTION}

The term ectodermal dysplasia (ED) was given by Weech and reported first by Thurman. ${ }^{[1]}$ National Foundation for ED has defined ED as a genetic disorder in which there are congenital birth defects of two or more ectodermal structures. ${ }^{[2]}$ The incidence rate is very low and it is estimated to be one in 1 lakh. ${ }^{[1]}$ It is a complex disease with more than 190 different clinical conditions and associated with various syndromes. ED can occur as early as the $6^{\text {th }}$ week of intrauterine life and during the first trimester. It is classified into two major types based on number and functionality of sweat glands: (1) Anhidrotic or hypohidrotic: Sweat glands

\begin{tabular}{|l|l|}
\hline Quick Response Code & Article Info: \\
\hline doi: 10.5866/2017.9.10191 \\
\hline $\begin{array}{l}\text { Received: 09-08-2017 } \\
\text { Revised: } 13-09-2017 \\
\text { Accepted: } 22-09-2017 \\
\text { Available Online: } 27-11-2017 \text { (www.nacd. } \\
\text { in)@ NAD, 2017 - All rights reserved }\end{array}$ \\
\hline
\end{tabular}

are significantly reduced or absent and called as Christ-Siemens-Touraine syndrome. (2) Hidrotic: Sweat glands are normal and called as Clouston's syndrome. Anhidrotic or hypohidrotic type is more common. ED is inherited as autosomal dominant or recessive or $\mathrm{X}$-linked recessive trait. Due to the complex mode of inheritance, it is mainly manifested in males and females are mostly carriers. Clinical features of ED are as follows: Onchodysplasia (nail dystrophy), hypotrichosis or alopecia (scanty, fine light hair on the scalp, and eyebrows), and palmoplantar hyperkeratosis. Other general features include: Asteatosis (absence of sebaceous glands), normal general mental development, dry skin, nail dystrophy, palmoplantar dyskeratosis, decreased sebaceous and nasal secretions, lack of dermal ridges, and fragile-appearing skin. Physical growth and psychomotor development are not affected.

Craniofacial features include raspy voice, depressed nasal bridge, hyperpigmentation of periorbital region, underdeveloped alveolar ridge, conical- or peg-shaped teeth, prominent lips, 
presence of neonatal teeth, and large low set ears. In oropharynx, high palatal arch and cleft palate may occur. Hypoplasia of intraoral accessory glands resulting in xerostomia, dry and cracked lips, prominent supraorbital ridge, frontal bossing, sunken cheeks, retruded appearance of the midface, total absence of teeth (anodontia), partial absence of teeth (hypodontia), reduced vertical dimension of face, malformed teeth, dry and pale oral mucosa, absence or reduced vermilion, and old appearance of face ${ }^{[3,4]}$ Management is mainly focused on counseling of parents and affected child for optimizing growth and normal psychosocial development, restoration of oral function along with esthetics, and prevention and treatment of hyperthermia. ED affects the social and psychological well-being of the patient. It also hampers the patient's self-image and speech in early childhood and leads to future behavioral changes in late teens. It has been reported that the child's self-image is completed by $4-5$ years. ${ }^{[5]}$ Therefore, the cosmetic and oral rehabilitative treatment should be intuited as early as possible. According to Pigno et al., initial prosthesis should be delivered before school age of the patient. ${ }^{[6]}$ Regarding the craniofacial features, the basic problem in ED patients is of missing teeth which lead to a cyclic chain of problems like reduced vertical dimension, deficient alveolar ridge and which leads to patient looking old and may have speech problem. Therefore, treatment of these patients is preferably replacement of missing teeth which will address most of the deformities. Other features can be symptomatically treated. Dental management of ED patients is a major challenge because of the fact that they can have complete anodontia, partial anodontia, malformed teeth, minimal or no alveolar ridge, dry and thin mucosa, and requiring dental treatment in early age, leading to noncompliance during treatment and acceptance of prosthesis. The case report emphasizes the dental management of two patients with hypohidrotic ED.

\section{CASE REPORTS}

\section{Case 1}

A 3-year-old male child came to us with parent complaining of noneruption of teeth. On clinical examination, the child had classical triad of hypohidrosis, hypotrichosis, and hypodontia. Along with this, the child had dry skin, sunken cheeks, depressed nasal bridge, protruded lips, prominent frontal bone, and supraorbital ridges [Figure 1]. Parents also informed that the child was intolerant to heat and they have to place wet, cold cloth, or towel to make him comfortable. The child also had speech defects. On intraoral examination: Only two conical-shaped teeth were present in the upper anterior region [Figure 2]. The lower jaw was completely edentulous. There was hardly any alveolar ridge present. There was no similar history in the family. Past medical history was insignificant. Orthopantogram (OPG) was done. OPG findings confirmed hypodontia with the presence of coneshaped primary incisors, and three tooth buds of permanent teeth (incisors and canine) and totally edentulous mandible without any tooth buds of permanent tooth. Height of alveolar ridge was minimal [Figure 3]. Based on the above findings,

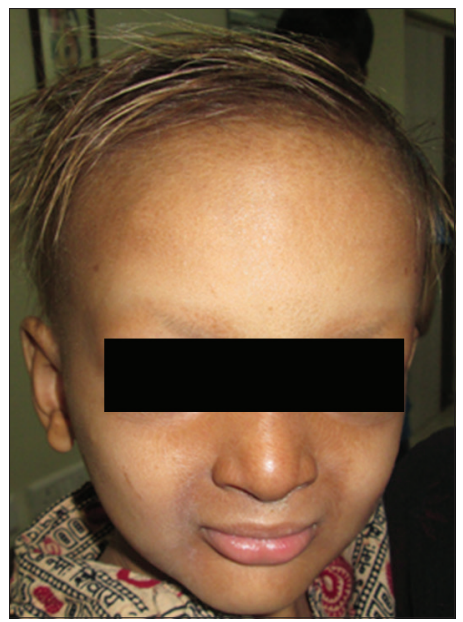

Figure 1: Clinical picture showing dry skin, sunken cheeks, depressed nasal bridge, protruded lips, prominent frontal bone, and supraorbital ridges



Figure 2: Intraoral examination reveals only two conicalshaped teeth in upper anterior region 
a diagnosis of hypohidrotic ED was made. The parents were explained about the condition. The child was decided to be rehabilitated with complete denture based on the age and OPG findings. Based on uncooperativeness and reluctance to accept the treatment and child displaying evidence of slight negativism, the child was graded "negative" according to the Frank 1 rating scale for behavior. "Tell show do" technique of behavior modulation was used to which the patient responded well and become progressively cooperative. All the routine steps required for denture fabrication with few modifications were followed: Primary impression was done with stock metal tray with polyvinyl siloxane putty impression material. Custom trays were made using acrylic and space was created for the two conical teeth which were present lingually and border molding was carried out using green stick material. Final impression was done with light body polyvinyl siloxane impression material and master casts were poured. Jaw relation was recorded after assessing esthetics and phonetics. During this, the two conical teeth were slightly trimmed as they were creating hindrance in jaw relation. Teeth arrangement was done such that there was less force directed over the ridges to prevent alveolar bone loss. For this, the teeth were reduced in width and height and cuspal inclines were reduced. Try in was done and dentures were acrylized and polished. Final insertion was done and post-insertion instructions were explained to the child and the parents.

Space was created for the two natural teeth which were present in the complete denture. The space was created such that it helps in retention of the denture. Denture retention adhesive (FIXON denture adhesive, ICPA) was prescribed initially to make the denture more comfortable and make the child to use it. Other instructions such as to maintain soft diet and cleaning instructions for the denture were given. The parents were also advised to remove the denture at night for healing of oral tissue. The child was recalled after 2 days and final adjustment was done for pressure spots. During follow-up, after 1 week, the behavior of child changed. His self-esteem improved along with socialization skills [Figure 4]. A follow-up protocol was given to the parents. The patient was recalled after 15 days, 3 and 6 months. Parents were also told that child will need rebasing or change of denture as the growth of the jaws continues. Therefore, they were advised a follow-up after every 6 months. The

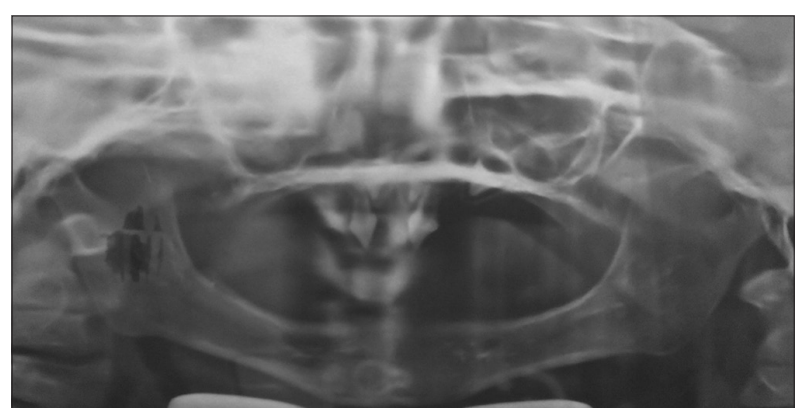

Figure 3: Orthopantogram showing hypodontia with the presence of cone-shaped primary incisors, and three tooth buds of permanent teeth (incisors and canine), and totally edentulous mandible without any tooth buds of permanent tooth

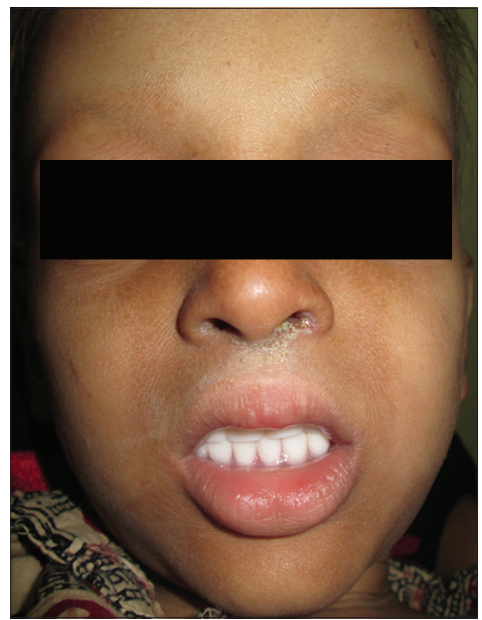

Figure 4: Post-operative picture showing denture with good retention and improved esthetics

patient was also referred to a speech therapist for speech evaluation and speech therapy.

\section{Case 2}

The second case was a 9-year-old male with complaint of missing teeth in his upper and lower jaws. On extraoral examination: This patient also had classical triad of hypohidrosis, hypotrichosis, and hypodontia along with prominent supraorbital ridge and forehead, sunken cheeks, depressed nasal bridge, and protruded lips. He was also intolerant to heat and speech problem. On intraoral examination: Only two conical-shaped teeth were present in the upper anterior region. The lower jaw was completely edentulous [Figure 5]. There was hardly any alveolar ridge present. There was no similar history in the family. Past medical history was insignificant. OPG was done. OPG findings confirmed hypodontia with the presence 
of cone-shaped incisors and totally edentulous mandible without any tooth buds of permanent tooth. Alveolar ridge was minimal in both the jaws [Figure 6]. Based on the above findings, a diagnosis of hypohidrotic ED was made. The treatment options were explained and it was decided the child would be rehabilitated with complete denture based on the age and OPG findings. This patient was very cooperative and the child was graded "definitely positive" according to the Frank 1 rating scale for behavior. All the routine steps required for denture fabrication with few modifications similar to Case 1 was done. Final insertion was done and postinsertion instructions were explained to the child and the parents [Figure 7].

Denture retention adhesive (FIXON denture adhesive, ICPA) was prescribed initially to make the denture more comfortable and make the child to use it. Other instructions and follow-up protocol were given similar to Case 1 and this patient was also referred for speech evaluation and therapy. During 1 week follow-up, remarkable change was seen in the psychosocial behavior and esthetics of the child. He was very comfortable and happy with the dentures. Improvement was seen in socialization skills and speech also.

\section{DISCUSSION}

It has been seen that a definitive classification of ED is not possible due to the fact that most of the syndromes have overlapping features. Various classifications have been proposed for ED. In 1982, Pinheiro and Freire-Maia proposed the first classification system for ED ${ }^{[7]}$ This classification was again updated in 1994 and 2001. In 2003, another classification is given by Lamartine based on the underlying pathophysiologic defect: (1) Cell-to-cell communication and signaling, (2) adhesion, (3) development, and (4) others. Similarly, in 2001, Priolo and Laganà reclassified the EDs into two main functional groups: (1) Defects in developmental regulation/epithelial-mesenchymal interaction and (2) defects in cytoskeleton maintenance and cell stability. Some ED may be mild, whereas others may be devastating. Sometimes, the diagnosis becomes difficult as the characteristic features are not obvious during birth, although during the neonatal period, there is extensive scaling of the skin and unexplained pyrexia. Etiology of ED as described by Freire-Maia is due to a development defect at embryonic level affecting the ectodermal tissues and structures formed from it. ${ }^{[8]}$ The mode is inheritance

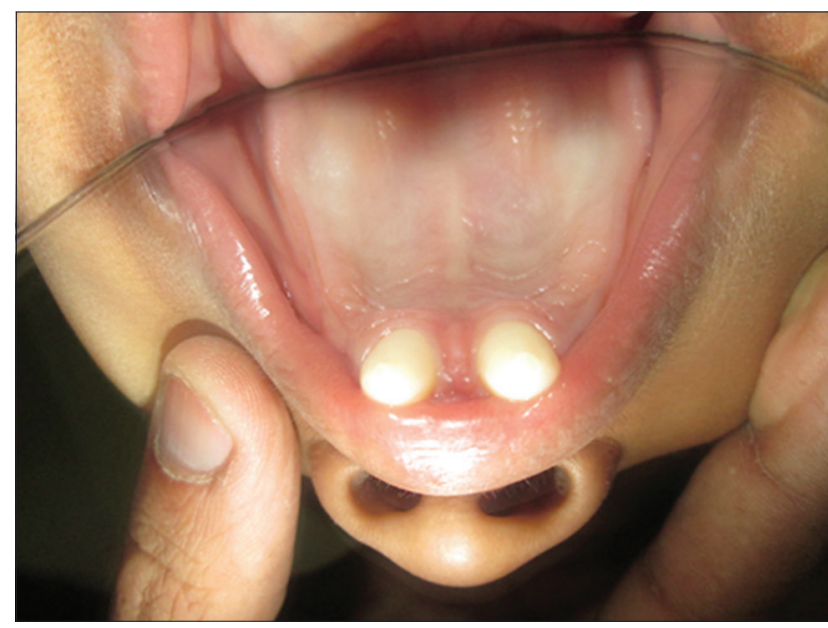

Figure 5: Clinical picture showing only two conical-shaped teeth in upper anterior region

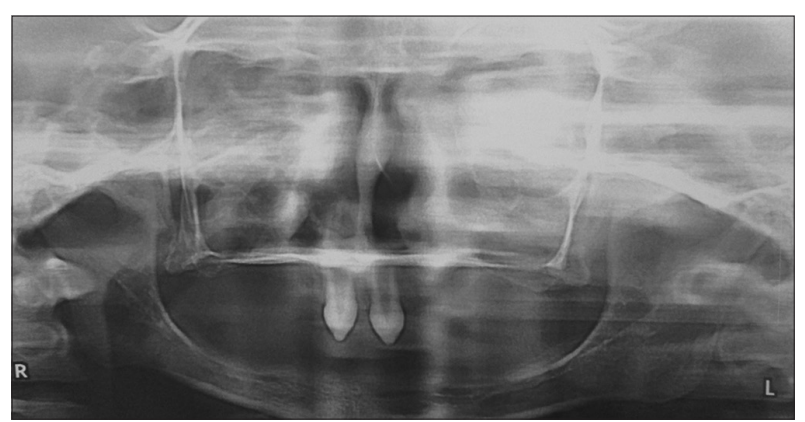

Figure 6: Orthopantogram reveal only two conical-shaped teeth in upper anterior region and the lower jaw was completely edentulous

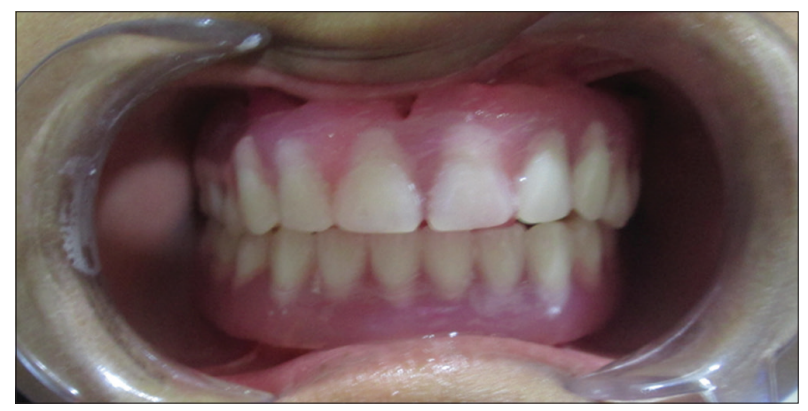

Figure 7: Post-operative picture showing final insertion of the denture

is both autosomal and X-linked. Genetic studies have shown the mutation of gene ectodysplasin-A and ectodysplasin-A receptor causes ED. Therefore, it is manifested in males and deformities occur only in $60-70 \%$ of females with much less intensity. This is explained by Lyons hypothesis. Females show minimal signs and symptoms restricted to 
hypodontia, minor ophthalmic diseases including impaired lacrimal gland function, glaucoma, aplastic or hypoplastic mammary glands, increased susceptibility to asthma, or eczema. The cells which are affected in ED are keratinocytes, leading to aberrations in development of hair, eccrine and apocrine glands, nails, sebaceous glands, lenses and conjunctiva of the eyes, anterior pituitary gland, ears, and teeth. Diagnosis of ED is basically based on clinical manifestations such as the distribution of sweat pores and their number, quantity, and quality of sweat produced, biochemical and structure characteristics of hair, biopsy of skin, and biochemical analysis of lacrimal secretions. Patients are immunodeficient with hypogammaglobulinemia and impaired lymphocyte proliferation and cellmediated immunity. Radiographs of feet and hand may demonstrate skeletal deformities. Differential diagnosis includes Werner syndrome, focal dermal hypoplasia, alopecia areata, aplasia cutis congenita, incontinentia pigmenti, dyskeratosis congenital, familial simple anhidrosis, Naegeli-FranceschettiJadassohn syndrome, and pachyonychia congenita. Treatment of ED patients is a very complex issue. Due to the presence of multiple abnormities and its mode of inheritance, it requires a team approach consisting of pediatrician, prosthodontist, dermatologist, otolaryngologist, speech therapist, and psychologist. The treatment plan has two goals which are the restoration of function and esthetics. Dental treatment should be started as early as possible because of the fact that hypodontia or anodontia of ED leads to abnormalities of behavior, esthetics, speech, and function. ED patients associated with cleft lip and palate requires intervention by maxillofacial and plastic surgeon. Therefore, we decided to rehabilitate our first case at the age of 3 years before the behavioral changes start. This will make the child adapt to the dentures and his esthetics and functions are restored before he starts school. Due to such a small age, the behavior modification techniques were used to make the child to have a positive attitude toward the dental treatment. For this child, as the growth advances, he will require multiple dental visits and follow-ups for proper adjustment of the dentures and for stability and retention. It has been seen that as the child grows, the denture replacement may be needed at least 3 times from early to late mixed dentition to permanent dentition. For the second case, again dentures were fabricated because the child was still in growth phase. He was also advised long-term follow-up The option for implant-supported prosthesis was not considered because of the fact that growth will cause implant movement and alveolar ridge was minimal and child will require some sort of bone grafting before the implants are placed. The main problems associated rehabilitation with complete denture include: Presence of few teeth may require modifications so that rather than causing hindrance they help in retention and stability, sometimes, they may lead to modifications in the denture for accommodation, recording vertical dimension in children is also difficult. Silverman has advised to include morphologic, physiologic phenomena, and functional activity to access the proper jaw relation which is applicable for all pediatric patients requiring complete dentures ${ }^{[9]}$ Another problem is difficult in the selection of tooth size and type. To reduce forces on the compromised ridges, small size tooth should be selected with reduced mesiodistal width and cusp ridges should be obtuse. Problems of retention and stability can be overcome with the help of following the proper steps of denture fabrication. We recommend primary, impression as well as secondary impression, should be taken with elastomeric impression materials for this. Elastomeric materials are not only easy to handle which is especially needed in pediatric patients but also helps in recording exact anatomical landmarks. We also recommend the use of denture adhesives during initial phases of denture wearing for pediatric patients this help in better compliance of the children. During the initial follow-up, a great change in the behavior was seen for both the patients. They seem to be more confident and a lot of improvement was seen on facial features restored vertical dimensions, lip positing was improved, and sunken cheek appearance was gone. It has also lead to improvement in speech and masticatory function. Patients with ED must have access to water and cool environment such as air-conditioned rooms. They can be advised to use wet T-shirts or cooling vests. Few studies have recommended that after growth phase is over for ED patients the removable dentures should be replaced with implant-supported complete denture. Advantages of this are better patient satisfaction and treatment experience along with improved mandibular function. Furthermore, better retention and adaptation are seen. ${ }^{[10]}$ Various studies have also highlighted the problems associated with the use of implants in ED patients such as early loss of implant failure, restricted 
growth of mandible, change in jaw relation over a period of time, and cost factor. Therefore, these case reports highlight the importance of accurate diagnosis and treatment plan should be modified as per the clinical findings and a team approach should be used for these patients. It also highlights the role of early dental treatment for these patients to restore function and normal psychosocial behavior.

\section{CONCLUSION}

Management of ED patients is a complex issue. It requires a team effort for overall management. Dental treatment should be individualized according to case. These patients require long-term follow-up until the growth period is over and may require complex procedures.

\section{REFERENCES}

1. Nunn JH, Carter NE, Gillgrass TJ, Hobson RS, Jepson NJ, Meechan JG, et al. The interdisciplinary management of hypodontia: Background and role of paediatric dentistry. $\mathrm{Br}$ Dent J 2003;194:245-51.

2. Hickey AJ, Vergo TJ Jr. Prosthetic treatment for patients with ectodermal dysplasia. J Prosthet Dent 2001;86:364-68.

3. Tarjan I, Gabris K, Rozsa N. Early prosthetic treatment of patients with ectodermal dysplasia: A clinical report. J Prosthet Dent 2005;93:419-24.

4. Vieira KA, Teixeira MS, Guirado CG, Gavião MB Prosthodontic treatment of hypohidrotic ectodermal dysplasia with complete anodontia: Case report. Quintessence Int 2007;38:75-80.

5. Guckes AD, Brahim JS, McCarthy GR, Rudy SF, Cooper LF. Using endosseous dental implants for patients with ectodermal dysplasia. J Am Dent Assoc 1991;122:59-62.

6. Pigno MA, Blackman RB, Cronin RJ, Cavazos E. Prosthodontic management of ectodermal dysplasia: A review of the literature. J Prosthet Dent 1996;76:541-5.

7. Pinheiro M, Freire-Maia N. The ectodermal dysplasias. Arch Dermatol 1982;118:215-6.

8. Itthagarun A, King NM. Ectodermal dysplasia: A review and case report. Quintessence Int 1997;28:595-602.

9. Silverman SI. Vertical dimension record:A three dimensional phenomenon. Part I. J Prosthet Dent 1985;53:420-5.

10. Yap AK, Klineberg I. Dental implants in patients with ectodermal dysplasia and tooth agenesis: A critical review of the literature. Int J Prosthodont 2009;22:268-76. 